\title{
Influence of Preparation Conditions on the Formation of Copper (II) Architectures with Pyrazine-2,3,5-tricarboxylic Acid
}

\author{
Feng-Qin Wang, ${ }^{*}$ Shu Lin, Ming-Lin Guo, Jun-Jian Xu, Xiao-Qing Wang, and Yong-Nan Zhao ${ }^{\dagger}$ \\ College of Environment and Chemical Engineering \& Tianjin Key Laboratory of Fiber Modification and Functional Fiber, \\ Tianjin Polytechnic University, Tianjin 300160, People's Republic of China. *E-mail: wfengqin@yahoo.com.cn \\ ${ }^{\dagger}$ College of Materials and Engineering \& Key Lab of Hollow Fiber Membrane Materials \& Membrane Process, \\ Tianjin Polytechnic University, Tianjin 300160, People's Republic of China \\ Received April 12, 2011, Accepted May 29, 2011
}

\begin{abstract}
Three new metal-organic copper(II) complexes, $\left[\mathrm{Cu}\left(\mathrm{H}_{2} \mathrm{PZTC}\right)_{2}\right]_{\mathrm{n}} \cdot 2 \mathrm{nH}_{2} \mathrm{O}(\mathbf{1}),\left[\mathrm{Cu}(\mathrm{HPZTC}) \cdot 2 \mathrm{H}_{2} \mathrm{O}\right]_{\mathrm{n}} \cdot 2 \mathrm{nH}_{2} \mathrm{O}$ (2), and $\mathrm{Cu}_{2}\left[(\mathrm{PZHD})(\mathrm{OH})\left(\mathrm{H}_{2} \mathrm{O}\right)_{2}\right]_{\mathrm{n}}(3)\left(\mathrm{H}_{3} \mathrm{PZTC}=\right.$ pyrazine-2,3,5-tricarboxylic acid, $\mathrm{PZHD}^{3-}=$ 2-hydroxypyrazine-3,5-dicarboxylate), have been synthesized from $\mathrm{Cu}(\mathrm{II}) / \mathrm{H}_{3} \mathrm{PZTC}$ system under different synthetic conditions, and characterized by single-crystal X-ray diffraction, elemental analysis, IR spectroscopy and thermogravimetric analysis. In complexes 1 and 2, $\mathrm{H}_{3}$ PZTC ligands loose one and two protons, which were transformed into $\mathrm{H}_{2} \mathrm{PZTC}^{-}$anion and $\mathrm{HPZTC}^{2-}$ dianion under different preparation condition, respectively. Furthermore, two ligands coordinate with $\mathrm{Cu}$ (II) cations in different modes, leading to the formation of the different chain structures. In complex 3, $\mathrm{H}_{3}$ PZTC ligand was converted into a new ligand-PZHD by in situ decarboxylation and hydroxylation under a higher $\mathrm{pH}$ value than that for complexes $\mathbf{1}$ and $\mathbf{2}$. PZHD ligands link the $\mathrm{Cu}(\mathrm{II})$ cations to form a 2D layer structure. These results demonstrate that the preparation conditions, including $\mathrm{pH}$ value and reaction temperature etc, play an important role in the construction of complexes based on $\mathrm{H}_{3}$ PZTC ligand.
\end{abstract}

Key Words : Pyrazine-2,3,5-tricarboxylic acid, Preparation condition, In situ reaction

\section{Introduction}

The metal-organic frameworks (MOFs) have received intensive interests, mostly motivated by their intriguing variety of architectures and potential applications in various areas such as catalysis, magnetism, gas storage, and luminescence, etc. ${ }^{1-12}$ The reported data have demonstrated that not only the selection of proper organic linkers and metal ions but also the synthetic conditions such as reaction temperatures and $\mathrm{pH}$ values et al., ${ }^{13-18}$ have a great influence on the structures and properties of the complexes.

Recently, multicarboxylate ligands have been usually selected as multifunctional organic linkers for the abundant coordination modes, and also for the ability to act as hydrogen bond acceptors or donors to assemble supramolecular structures. ${ }^{19,20}$ Pyrazine-2,3,5-tricarboxylic acid $\left(\mathrm{H}_{3}\right.$ PZTC) is one of the above mentioned multicarboxylate ligands and therefore a multifunctional organic linker. It has several remarkable features as follows: (1) It contains three carboxylate groups and two nitrogen atoms, which can coordinate to metal ions in various coordination modes, and assemble into novel and fantastic structures. (2) The three carboxylate groups are very sensitive to the acidity/basicity of the reaction system, which can be partially or fully deprotonated under different $\mathrm{pH}$ values, and then assembly into different architectures. (3) Moreover, the coordination features of this ligand and the dependence of its complex structures on various preparation conditions have been little investigated to date. ${ }^{21,22}$
Therefore, we selected the $\mathrm{H}_{3}$ PZTC as organic ligand and synthesized three new copper(II) complexes, $\left[\mathrm{Cu}\left(\mathrm{H}_{2} \mathrm{PZTC}\right)_{2}\right]_{n}$. $2 \mathrm{nH}_{2} \mathrm{O}(\mathbf{1}),\left[\mathrm{Cu}(\mathrm{HPZTC}) \cdot 2 \mathrm{H}_{2} \mathrm{O}\right]_{\mathrm{n}} \cdot 2 \mathrm{nH}_{2} \mathrm{O}$, (2) and $\mathrm{Cu}_{2}$ [(PZHD)$\left.(\mathrm{OH})\left(\mathrm{H}_{2} \mathrm{O}\right)_{2}\right]_{\mathrm{n}}(3)$ based on $\mathrm{Cu} / \mathrm{H}_{3}$ PZTC system under different synthetic conditions. In this paper, we report the syntheses, crystal structure and thermal properties of the title complexes.

\section{Experimental Section}

Materials and General Methods. Pyrazine-2,3,5-tricarboxylic acid dihydrate was synthesized according to the literatures. ${ }^{23-25}$ All the other reagents were used as received without further purification. The $\mathrm{C}, \mathrm{H}, \mathrm{N}$ microanalyses were carried out on a Vario EL elemental analyzer. The IR spectrum was recorded on a Nicolet Avatar 360 FT-IR spectrometer by using the $\mathrm{KBr}$ pellet technique. The TG experiment for the complex was performed using A NETZSCH STA $409 \mathrm{PG} / \mathrm{PC}$ thermogravimetric analyzer under $\mathrm{N}_{2}$ atmosphere. The heating rate was $10{ }^{\circ} \mathrm{C} \min ^{-1}$ from ambient temperature to $1000^{\circ} \mathrm{C}$ with a flow rate of 20 $\mathrm{mL} \cdot \mathrm{min}^{-1}$.

Crystal Structure Determinations. The single-crystal Xray data collections for complexes 1-3 were performed on a Bruker SMART 1000 CCD area detector diffractometer with graphite-monochromated Mo K $\alpha$ radiation $(\lambda=0.71073 \AA)$. The raw data integrated into SHELX-format reflection files and semi-empirical absorption corrections were applied by using the SADABS program. ${ }^{26}$ The structures were solved 
Table 1. Crystal data and structure refinement parameters for complexes 1-3

\begin{tabular}{llll}
\hline Complex & $\mathbf{1}$ & $\mathbf{2}$ & $\mathbf{3}$ \\
\hline Chemical formula & $\mathrm{C}_{14} \mathrm{H}_{10} \mathrm{CuN}_{4} \mathrm{O}_{14} \mathrm{C}_{7} \mathrm{H}_{10} \mathrm{CuN}_{2} \mathrm{O}_{10} \mathrm{C}_{6} \mathrm{H}_{6} \mathrm{Cu}_{2} \mathrm{~N}_{2} \mathrm{O}_{8}$ \\
Formula weight & 521.80 & 345.71 & 361.21 \\
Temperature $[\mathrm{K}]$ & $133(2)$ & $133(2)$ & $133(2)$ \\
Crystal system & Triclinic & Monoclinic & Monoclinic \\
Space group & $\mathrm{P}_{-1}$ & $\mathrm{P}_{1} / \mathrm{c}$ & $\mathrm{P}{ }_{1}$ \\
$a(\AA)$ & $6.728(13)$ & $6.594(13)$ & $9.222(18)$ \\
$b(\AA)$ & $7.854(16)$ & $12.493(3)$ & $6.191(12)$ \\
$c(\AA)$ & $9.638(19)$ & $14.112(4)$ & $9.470(19)$ \\
$\alpha\left(^{\circ}\right)$ & $83.12(3)$ & 90 & 90 \\
$\beta\left({ }^{\circ}\right)$ & $83.79(3)$ & $107.30(3)$ & $116.99(3)$ \\
$\gamma\left({ }^{\circ}\right)$ & $77.95(3)$ & 90 & 90 \\
$V\left(\AA^{3}\right)$ & $492.68(17)$ & $1109.9(5)$ & $481.8(2)$ \\
$Z$ & 1 & 4 & 2 \\
$\mathrm{~F}(000)$ & 263 & 700 & 356 \\
$\rho\left[\mathrm{mg} \mathrm{m}^{-3}\right]$ & 1.759 & 2.069 & 2.490 \\
$\mu\left[\mathrm{mm}{ }^{-1}\right]$ & 1.194 & 2.029 & 4.455 \\
$\theta$ range $($ deg $)$ & $2.67-27.04$ & $3.02-27.00$ & $2.41-27.49$ \\
Reflections collected 3359 & 7376 & 3349 \\
Total independent, & $2134,0.0301$ & $2411,0.0361$ & $1690,0.0528$ \\
Rint & & & \\
Goodness-of-fit on $\mathrm{F}^{2} 0.996$ & 1.053 & 1.052 \\
Flack parameter & & & $0.02(2)$ \\
$\mathrm{R}$ indices $[\mathrm{I}>2 \sigma(I)]$ & $\mathrm{R} 1=0.0311$ & $\mathrm{R} 1=0.0313$, & $\mathrm{R} 1=0.0344$, \\
& $\mathrm{wR} 2=0.0730$ & $\mathrm{wR} 2=0.0736$ & wR2 $=0.0727$ \\
\hline & & &
\end{tabular}

by direct methods ${ }^{27}$ and refined by full-matrix least squares on $\mathrm{F}^{2}$ by using SHELXS 97 and SHELXL 97 programs, respectively. ${ }^{27,28}$ All non-hydrogen atoms were refined anisotropically. The hydrogen atoms were generated geometrically and treated by a mixture of independent and constrained refinement. The crystallographic data for complexes 1-3 are listed in Table 1. Selected bond lengths $(\AA)$ and angles $\left({ }^{\circ}\right)$ are listed in Table 2 and 3. The details of the hydrogen bonds for the complexes are listed in Table 4.

Synthesis of $\left[\mathbf{C u}\left(\mathbf{H}_{2} \text { PZTC }\right)_{2}\right]_{\mathbf{n}} \cdot \mathbf{2} \mathbf{n H}_{2} \mathrm{O}(\mathbf{1}) \cdot \mathbf{C u}\left(\mathrm{NO}_{3}\right)_{2} \cdot 2 \mathrm{H}_{2} \mathrm{O}$ $(0.15 \mathrm{~g}, 6.2 \mathrm{mmol})$ was added into $15 \mathrm{~mL}$ aqueous solution of $\mathrm{H}_{3}$ PZTC ( $\left.0.20 \mathrm{~g}, 8 \mathrm{mmol}\right)$ under vigorous stirring over a period of $20 \mathrm{~min}$. The solution was filtered and maintained at room temperature for crystallization by slow evaporation. After two weeks, the blue block-like crystals of 1 were obtained. Crystals were isolated by filtration and air-dried. Yield: $64.3 \%$. Anal. Calc. for $\mathrm{C}_{14} \mathrm{H}_{10} \mathrm{CuN}_{4} \mathrm{O}_{14}$ : C, 37.56; $\mathrm{H}$, $1.92 ; \mathrm{N}, 10.73$. Found: C, 36.93; H, 2.27; N, 11.20\%. IR $\left(\mathrm{KBr}\right.$ pellet, $\left.\mathrm{cm}^{-1}\right)$ : 3468(b), 1658(s), 1430(m), 1360(m), 1312(s), 1180(s), 1108(m), 850(m), 790(m), 724(w).

Synthesis of $\left[\mathbf{C u}(\mathrm{HPZTC}) \cdot \mathbf{2} \mathrm{H}_{2} \mathrm{O}\right]_{\mathbf{n}} \cdot \mathbf{2} \mathbf{n H}_{2} \mathrm{O}$ (2). A mixture of $\mathrm{H}_{3} \mathrm{PZTC}(0.050 \mathrm{~g}, 0.2 \mathrm{mmol}), \mathrm{Cu}\left(\mathrm{NO}_{3}\right)_{2} \cdot 2 \mathrm{H}_{2} \mathrm{O}(0.072 \mathrm{~g}$, $0.3 \mathrm{mmol}), \mathrm{NaN}_{3}(0.013 \mathrm{~g}, 0.2 \mathrm{mmol}), \mathrm{NaOH}(0.15 \mathrm{~mL}$, $\left.0.65 \mathrm{~mol} \cdot \mathrm{L}^{-1}\right)$, and $\mathrm{H}_{2} \mathrm{O}(8 \mathrm{~mL})$ was put into a $25 \mathrm{~mL}$ stainless steel bomb and heated at $130{ }^{\circ} \mathrm{C}$ for 4 days, then cooled slowly to room temperature. Blue-green crystals of $\mathbf{2}$ were obtained by filtration. Yield: $72.2 \%$. Anal. Calc. for $\mathrm{C}_{7} \mathrm{H}_{10} \mathrm{CuN}_{2} \mathrm{O}_{10}$ : C, 24.29; H, 2.89; N, 8.10. Found: C, 23.84;
Table 2. Selected bond lengths $(\AA)$ and angles $\left({ }^{\circ}\right)$ for complexes 1 and 2

\begin{tabular}{|c|c|c|c|}
\hline \multicolumn{4}{|l|}{ Complex 1} \\
\hline $\mathrm{Cu}(1)-\mathrm{O}(1)$ & $1.9543(15)$ & $\mathrm{Cu}(1)-\mathrm{N}(1)$ & $1.9931(18)$ \\
\hline $\mathrm{Cu}(1)-\mathrm{O}(4)$ & $2.3978(15)$ & $\mathrm{O}(1)-\mathrm{Cu}(1)-\mathrm{O}(1) \# 1$ & 180.0 \\
\hline $\mathrm{O}(1)-\mathrm{Cu}(1)-\mathrm{N}(1)$ & $83.87(7)$ & $\mathrm{O}(1) \# 1-\mathrm{Cu}(1)-\mathrm{N}(1)$ & $96.13(7)$ \\
\hline $\mathrm{O}(1)-\mathrm{Cu}(1)-\mathrm{N}(1) \# 1$ & $96.13(7)$ & \multicolumn{2}{|c|}{$\mathrm{O}(1) \# 1-\mathrm{Cu}(1)-\mathrm{N}(1) \# 183.87(7)$} \\
\hline $\mathrm{N}(1) \# 1-\mathrm{Cu}(1)-\mathrm{N}(1)$ & $180.00(1)$ & $\mathrm{O}(1)-\mathrm{Cu}(1)-\mathrm{O}(4) \# 2$ & $88.76(6)$ \\
\hline $\mathrm{O}(1) \# 1-\mathrm{Cu}(1)-\mathrm{O}(4) \# 2$ & $91.24(6)$ & \multicolumn{2}{|c|}{$\mathrm{N}(1) \# 1-\mathrm{Cu}(1)-\mathrm{O}(4) \# 291.79(6)$} \\
\hline $\mathrm{N}(1)-\mathrm{Cu}(1)-\mathrm{O}(4) \# 2$ & $88.21(6)$ & \multicolumn{2}{|c|}{$\mathrm{O}(4) \# 2-\mathrm{Cu}(1)-\mathrm{O}(4) \# 3180.0$} \\
\hline $\mathrm{O}(1)-\mathrm{Cu}(1)-\mathrm{O}(4) \# 3$ & $91.24(6)$ & \multicolumn{2}{|c|}{$\mathrm{O}(1) \# 1-\mathrm{Cu}(1)-\mathrm{O}(4) \# 388.76(6)$} \\
\hline $\mathrm{N}(1)-\mathrm{Cu}(1)-\mathrm{O}(4) \# 3$ & $91.79(7)$ & \multicolumn{2}{|c|}{$\mathrm{N}(1) \# 1-\mathrm{Cu}(1)-\mathrm{O}(4) \# 388.21(6)$} \\
\hline \multicolumn{4}{|l|}{ Complex 2} \\
\hline $\mathrm{C} u(1)-\mathrm{O}(7)$ & $2.0271(16)$ & $\mathrm{Cu}(1)-\mathrm{O}(8)$ & $2.0329(16)$ \\
\hline $\mathrm{Cu}(1)-\mathrm{O}(3) \# 1$ & $2.1939(17)$ & $\mathrm{Cu}(1)-\mathrm{O}(1)$ & $2.2179(17)$ \\
\hline $\mathrm{Cu}(1)-\mathrm{N}(1)$ & $2.241(2)$ & $\mathrm{Cu}(1)-\mathrm{O}(6)$ & $2.3012(17)$ \\
\hline $\mathrm{Cu}(1)-\mathrm{N}(2) \# 1$ & $2.397(2)$ & & \\
\hline $\mathrm{O}(7)-\mathrm{Cu}(1)-\mathrm{O}(8)$ & $173.30(7)$ & $\mathrm{O}(7)-\mathrm{Cu}(1)-\mathrm{O}(3) \# 1$ & $95.63(7)$ \\
\hline $\mathrm{O}(8)-\mathrm{Cu}(1)-\mathrm{O}(3) \# 1$ & $88.98(6)$ & $\mathrm{O}(7)-\mathrm{Cu}(1)-\mathrm{O}(1)$ & $92.14(6)$ \\
\hline $\mathrm{O}(8)-\mathrm{Cu}(1)-\mathrm{O}(1)$ & $93.73(6)$ & $\mathrm{O}(3) \# 1-\mathrm{Cu}(1)-\mathrm{O}(1)$ & $75.48(6)$ \\
\hline $\mathrm{O}(7)-\mathrm{Cu}(1)-\mathrm{N}(1)$ & $89.13(7)$ & $\mathrm{O}(8)-\mathrm{Cu}(1)-\mathrm{N}(1)$ & $89.60(7)$ \\
\hline $\mathrm{O}(3) \# 1-\mathrm{Cu}(1)-\mathrm{N}(1)$ & $147.44(7)$ & $\mathrm{O}(1)-\mathrm{Cu}(1)-\mathrm{N}(1)$ & $72.16(6)$ \\
\hline $\mathrm{O}(7)-\mathrm{Cu}(1)-\mathrm{O}(6)$ & $87.33(6)$ & $\mathrm{O}(8)-\mathrm{Cu}(1)-\mathrm{O}(6)$ & $86.08(6)$ \\
\hline $\mathrm{O}(3) \# 1-\mathrm{Cu}(1)-\mathrm{O}(6)$ & $144.03(6)$ & $\mathrm{O}(1)-\mathrm{Cu}(1)-\mathrm{O}(6)$ & $140.36(6)$ \\
\hline $\mathrm{N}(1)-\mathrm{Cu}(1)-\mathrm{O}(6)$ & $68.21(6)$ & $\mathrm{O}(7)-\mathrm{Cu}(1)-\mathrm{N}(2) \# 1$ & $86.95(7)$ \\
\hline $\mathrm{O}(8)-\mathrm{Cu}(1)-\mathrm{N}(2) \# 1$ & $90.14(7)$ & $\mathrm{O}(3) \# 1-\mathrm{Cu}(1)-\mathrm{N}(2) \#$ & $169.35(6)$ \\
\hline $\mathrm{O}(1)-\mathrm{Cu}(1)-\mathrm{N}(2) \# 1$ & $144.53(7)$ & $\mathrm{N}(1)-\mathrm{Cu}(1)-\mathrm{N}(2) \# 1$ & $143.19(7)$ \\
\hline $\mathrm{O}(6)-\mathrm{Cu}(1)-\mathrm{N}(2) \# 1$ & $75.06(6)$ & & \\
\hline
\end{tabular}

Symmetry transformations used to generate equivalent atoms. For 1: \#1 $-\mathrm{x}+1,-\mathrm{y}+1,-\mathrm{z}+1 \# 2 \mathrm{x}+1, \mathrm{y}, \mathrm{z} \# 3-\mathrm{x},-\mathrm{y}+1,-\mathrm{z}+1$, For 2 : \#1 $\mathrm{x},-\mathrm{y}+1 / 2$, $\mathrm{z}-1 / 2$

Table 3. Selected bond lengths $(\AA)$ and angles $\left({ }^{\circ}\right)$ for complex 3

\begin{tabular}{llll}
\hline $\mathrm{Complex} \mathrm{3}$ & & & \\
\hline $\mathrm{Cu}(1)-\mathrm{O}(7)$ & $1.916(4)$ & $\mathrm{Cu}(1)-\mathrm{O}(5)$ & $1.921(4)$ \\
$\mathrm{Cu}(1)-\mathrm{O}(1)$ & $1.983(3)$ & $\mathrm{Cu}(1)-\mathrm{N}(1) \# 1$ & $1.994(4)$ \\
$\mathrm{Cu}(1)-\mathrm{O}(8)$ & $2.493(4)$ & $\mathrm{Cu}(1)-\mathrm{O}(5) \# 1$ & $2.706(4)$ \\
$\mathrm{Cu}(2)-\mathrm{O}(7)$ & $1.937(3)$ & $\mathrm{Cu}(2)-\mathrm{O}(6)$ & $1.957(4)$ \\
$\mathrm{Cu}(2)-\mathrm{N}(2) \# 2$ & $2.014(4)$ & $\mathrm{Cu}(2)-\mathrm{O}(1)$ & $2.058(4)$ \\
$\mathrm{Cu}(2)-\mathrm{O}(2) \# 2$ & $2.382(4)$ & $\mathrm{Cu}(2)-\mathrm{O}(3) \# 2$ & $2.383(4)$ \\
$\mathrm{O}(7)-\mathrm{Cu}(1)-\mathrm{O}(5)$ & $170.38(15)$ & $\mathrm{O}(7)-\mathrm{Cu}(1)-\mathrm{O}(1)$ & $79.69(14)$ \\
$\mathrm{O}(5)-\mathrm{Cu}(1)-\mathrm{O}(1)$ & $92.41(14)$ & $\mathrm{O}(7)-\mathrm{Cu}(1)-\mathrm{N}(1) \# 1$ & $98.58(17)$ \\
$\mathrm{O}(5)-\mathrm{Cu}(1)-\mathrm{N}(1) \# 1$ & $88.58(17)$ & $\mathrm{O}(1)-\mathrm{Cu}(1)-\mathrm{N}(1) \# 1$ & $172.8(2)$ \\
$\mathrm{O}(7)-\mathrm{Cu}(2)-\mathrm{O}(6)$ & $92.06(15)$ & $\mathrm{O}(7)-\mathrm{Cu}(2)-\mathrm{N}(2) \# 2$ & $175.35(18)$ \\
$\mathrm{O}(6)-\mathrm{Cu}(2)-\mathrm{N}(2) \# 2$ & $92.41(16)$ & $\mathrm{O}(7)-\mathrm{Cu}(2)-\mathrm{O}(1)$ & $77.38(13)$ \\
$\mathrm{O}(6)-\mathrm{Cu}(2)-\mathrm{O}(1)$ & $169.18(15)$ & $\mathrm{N}(2) \# 2-\mathrm{Cu}(2)-\mathrm{O}(1)$ & $98.20(16)$ \\
$\mathrm{O}(7)-\mathrm{Cu}(2)-\mathrm{O}(2) \# 2$ & $106.56(13)$ & $\mathrm{O}(6)-\mathrm{Cu}(2)-\mathrm{O}(2) \# 2$ & $93.09(16)$ \\
$\mathrm{N}(2) \# 2-\mathrm{Cu}(2)-\mathrm{O}(2) \# 2$ & $74.47(15)$ & $\mathrm{O}(1)-\mathrm{Cu}(2)-\mathrm{O}(2) \# 2$ & $87.74(15)$ \\
$\mathrm{O}(7)-\mathrm{Cu}(2)-\mathrm{O}(3) \# 2$ & $102.55(14)$ & $\mathrm{O}(6)-\mathrm{Cu}(2)-\mathrm{O}(3) \# 2$ & $90.31(16)$ \\
$\mathrm{N}(2) \# 2-\mathrm{Cu}(2)-\mathrm{O}(3) \# 2$ 76.15(15) & $\mathrm{O}(1)-\mathrm{Cu}(2)-\mathrm{O}(3) \# 2$ & $94.27(16)$ \\
$\mathrm{O}(2) \# 2-\mathrm{Cu}(2)-\mathrm{O}(3) \# 2$ & $150.54(11)$ & $\mathrm{O} 8-\mathrm{Cu} 1-\mathrm{O}$ (2) 1 & $155.41(13)$ \\
$\mathrm{O} 8-\mathrm{Cu} 1-\mathrm{O} 5$ & $88.19(13)$ & $\mathrm{O} 8-\mathrm{Cu} 1-\mathrm{N} 1 \# 1$ & $100.95(13)$ \\
\hline
\end{tabular}

Symmetry transformations used to generate equivalent atoms. \#1 $-\mathrm{x}$, $\mathrm{y}+1 / 2,-\mathrm{z}+1 \# 2-\mathrm{x}+1, \mathrm{y}+1 / 2,-\mathrm{z}+1$. 
Table 4. Hydrogen bonds geometries $\left(\AA,^{\circ}\right)$ for complexes 1-3

\begin{tabular}{|c|c|c|c|c|c|}
\hline $\mathrm{D}-\mathrm{H} \cdots \mathrm{A}$ & $\mathrm{d}(\mathrm{D}-\mathrm{H})$ & $\mathrm{d}(\mathrm{H} \cdots \mathrm{A})$ & $(\mathrm{D} \cdots \mathrm{A})$ & $\angle \mathrm{DHA}$ & Symmetry code \\
\hline \multicolumn{6}{|l|}{ Complex 1} \\
\hline $\mathrm{O}(3)-\mathrm{H}(3) \cdots \mathrm{O}(7)$ & 0.82 & 1.72 & $2.529(2)$ & $169.1(2)$ & {$[-1+\mathrm{x}, \mathrm{y}+1, \mathrm{z}]$} \\
\hline $\mathrm{O}(6)-\mathrm{H}(6) \cdots \mathrm{O}(2)$ & 0.82 & 1.74 & $2.554(2)$ & $171.9(2)$ & {$[\mathrm{x}, \mathrm{y}, \mathrm{z}+1]$} \\
\hline $\mathrm{O}(7)-\mathrm{H}(7 \mathrm{~A}) \cdots \mathrm{N}(2)$ & 0.85 & 2.28 & $3.073(2)$ & $155.6(2)$ & {$[-\mathrm{x},-\mathrm{y}+1,1-\mathrm{z}]$} \\
\hline $\mathrm{O}(7)-\mathrm{H}(7 \mathrm{~A}) \cdots \mathrm{O}(5)$ & 0.85 & 2.41 & $3.044(2)$ & $132.2(2)$ & {$[-\mathrm{x},-\mathrm{y}+1,1-\mathrm{z}]$} \\
\hline $\mathrm{O}(7)-\mathrm{H}(7 \mathrm{~B}) \cdots \mathrm{O}(5)$ & 0.85 & 2.05 & $2.894(3)$ & $168.3(2)$ & {$[\mathrm{x}+1, \mathrm{y}-1,-1+\mathrm{z}]$} \\
\hline \multicolumn{6}{|l|}{ Complex 2} \\
\hline $\mathrm{O}(5)-\mathrm{H}(5) \cdots \mathrm{O}(4)$ & 0.90 & 1.52 & $2.399(2)$ & $168(5)$ & {$[\mathrm{x}, \mathrm{y}, \mathrm{z}]$} \\
\hline $\mathrm{O}(7)-\mathrm{H}(7 \mathrm{~A}) \cdots \mathrm{O}(10)$ & 0.85 & 1.92 & $2.686(2)$ & $148.8(2)$ & {$[-\mathrm{x}+2, \mathrm{y}-1 / 2,-\mathrm{z}+3 / 2]$} \\
\hline $\mathrm{O}(7)-\mathrm{H}(7 \mathrm{~A}) \cdots \mathrm{O}(6)$ & 0.85 & 2.53 & $2.995(2)$ & $115.2(2)$ & {$[\mathrm{x}, \mathrm{y}, \mathrm{z}]$} \\
\hline $\mathrm{O}(7)-\mathrm{H}(7 \mathrm{~B}) \cdots \mathrm{O}(3)$ & 0.85 & 2.26 & $2.881(2)$ & $130.1(2)$ & {$[-\mathrm{x}+2, \mathrm{y}+1 / 2,-\mathrm{z}+3 / 2]$} \\
\hline $\mathrm{O}(7)-\mathrm{H}(7 \mathrm{~B}) \cdots \mathrm{O}(8)$ & 0.85 & 2.33 & $3.015(2)$ & $138.7(2)$ & {$[\mathrm{x}+1, \mathrm{y}, \mathrm{z}]$} \\
\hline $\mathrm{O}(8)-\mathrm{H}(8 \mathrm{~A}) \cdots \mathrm{O}(1)$ & 0.86 & 1.90 & $2.729(2)$ & $163.2(2)$ & {$[-\mathrm{x}+1,-\mathrm{y}+1,-\mathrm{z}+1]$} \\
\hline $\mathrm{O}(8)-\mathrm{H}(8 \mathrm{~B}) \cdots \mathrm{O}(9)$ & 0.85 & 1.91 & $2.684(2)$ & $149.7(2)$ & {$[\mathrm{x},-\mathrm{y}+1 / 2, \mathrm{z}-1 / 2]$} \\
\hline $\mathrm{O}(9)-\mathrm{H}(9 \mathrm{~A}) \cdots \mathrm{O}(2)$ & 0.86 & 1.91 & $2.757(2)$ & $169.0(2)$ & {$[-\mathrm{x}+1, \mathrm{y}-1 / 2,-\mathrm{z}+3 / 2]$} \\
\hline $\mathrm{O}(9)-\mathrm{H}(9 \mathrm{~B}) \cdots \mathrm{O}(4)$ & 0.85 & 2.03 & $2.824(2)$ & $154.9(2)$ & {$[-\mathrm{x}+1, \mathrm{y}+1 / 2,-\mathrm{z}+3 / 2]$} \\
\hline $\mathrm{O}(10)-\mathrm{H}(10 \mathrm{~A}) \cdots \mathrm{O}(2)$ & 0.86 & 2.01 & $2.816(2)$ & $155.8(2)$ & {$[-\mathrm{x}+1, \mathrm{y}-1 / 2,-\mathrm{z}+3 / 2]$} \\
\hline $\mathrm{O}(10)-\mathrm{H}(10 \mathrm{~B}) \cdots \mathrm{O}(5)$ & 0.85 & 2.06 & $2.899(2)$ & $167.5(2)$ & {$[\mathrm{x},-\mathrm{y}+1 / 2, \mathrm{z}+1 / 2]$} \\
\hline \multicolumn{6}{|l|}{ Complex $\mathbf{3}$} \\
\hline $\mathrm{O}(6)-\mathrm{H}(6 \mathrm{~A}) \cdots \mathrm{O}(4)$ & 0.85 & 1.73 & $2.567(5)$ & $170.0(2)$ & {$[-\mathrm{x}+1, \mathrm{y}+3 / 2,-\mathrm{z}+1]$} \\
\hline $\mathrm{O}(6)-\mathrm{H}(6 \mathrm{~B}) \cdots \mathrm{O}(8)$ & 0.85 & 2.16 & $2.700(5)$ & $121.2(2)$ & {$[-\mathrm{x}+1, \mathrm{y}+1 / 2,-\mathrm{z}+1]$} \\
\hline $\mathrm{O}(7)-\mathrm{H}(7 \mathrm{~A}) \cdots \mathrm{O}(3)$ & 0.85 & 2.20 & $3.035(5)$ & $169.3(2)$ & {$[\mathrm{x}, \mathrm{y}+1, \mathrm{z}+1]$} \\
\hline $\mathrm{O}(8)-\mathrm{H}(8 \mathrm{~A}) \cdots \mathrm{O}(3)$ & 0.84 & 1.96 & $2.804(5)$ & $177.4(2)$ & {$[-\mathrm{x}+1, \mathrm{y}+1 / 2,-\mathrm{z}+1]$} \\
\hline $\mathrm{O}(8)-\mathrm{H}(8 \mathrm{~B}) \cdots \mathrm{O}(7)$ & 0.85 & 2.04 & $2.886(6)$ & $172.6(2)$ & {$[\mathrm{x}, \mathrm{y}-1, \mathrm{z}]$} \\
\hline
\end{tabular}

H, 2.47; N, 7.83\%. IR (KBr pellet, $\mathrm{cm}^{-1}$ ): 2960(b), 1668(s), $1509(\mathrm{~m}), 1446(\mathrm{~m}), 1387(\mathrm{~s}), 1348(\mathrm{~s}), 1189(\mathrm{~m}), 1140(\mathrm{~m})$, 1060(w), 928(w), 792(w), 711(w).

Synthesis of $\left[\mathrm{Cu}_{2}(\mathrm{PZHD})(\mathrm{OH})\left(\mathrm{H}_{2} \mathrm{O}\right)_{2}\right]_{\mathbf{n}}$ (3). A mixture of $\mathrm{H}_{3} \mathrm{PZTC}(0.025 \mathrm{~g}, 0.1 \mathrm{mmol}), \mathrm{Cu}\left(\mathrm{NO}_{3}\right)_{2} \cdot 2 \mathrm{H}_{2} \mathrm{O}(0.048 \mathrm{~g}$, $0.2 \mathrm{mmol}), \mathrm{NaN}_{3}(0.013 \mathrm{~g}, 0.2 \mathrm{mmol}), \mathrm{NaOH}(0.15 \mathrm{~mL}$, $\left.0.65 \mathrm{~mol} \cdot \mathrm{L}^{-1}\right)$, and $\mathrm{H}_{2} \mathrm{O}(10 \mathrm{~mL})$ was put into a $25 \mathrm{~mL}$ stainless steel bomb and heated at $130{ }^{\circ} \mathrm{C}$ for 4 days, then cooled slowly to room temperature. Blue-green crystals of $\mathbf{3}$ were obtained by filtration. Yield: $54.2 \%$. Anal. Calc. for $\mathrm{C}_{6} \mathrm{H}_{6} \mathrm{Cu}_{2} \mathrm{~N}_{2} \mathrm{O}_{8}$ : C, 19.93; H, 1.66; N, 7.76. Found: C, 19.66;
H, 2.15; N, 8.14\%. IR (KBr pellet, $\left.\mathrm{cm}^{-1}\right)$ : 3365(b), 1665(s), 1584(m), 1508(s), 1382(s), 1314(m), 1201(s), 1126(w), 973(w), 824(m), 712(w).

\section{Results and Discussion}

Syntheses. As shown in Scheme 1, complexes 1, 2 and 3 were obtained from the $\mathrm{Cu}(\mathrm{II}) / \mathrm{H}_{3} \mathrm{PZTC}$ system under different synthetic conditions. In the routine condition, complex 1 was prepared by refluxing the aqueous solution of $\mathrm{Cu}\left(\mathrm{NO}_{3}\right)_{2} \cdot 2 \mathrm{H}_{2} \mathrm{O}$ and $\mathrm{H}_{3} \mathrm{PZTC}$ with the $\mathrm{pH}$ value of 1.8 . In

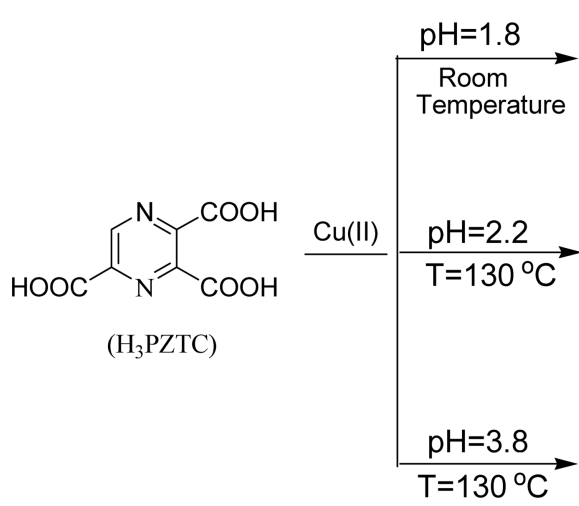

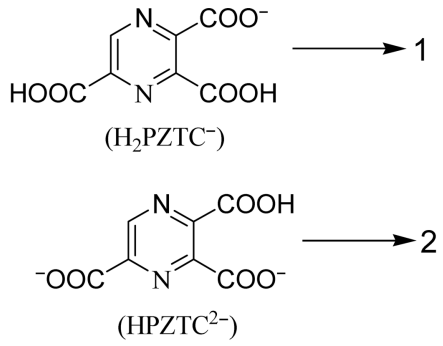

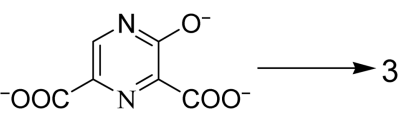

$\left(\mathrm{PZHD}^{3-}\right)$

Scheme 1. Simplified routes of syntheses for complexes 1-3. 
the complex, only one carboxylate group of $\mathrm{H}_{3} \mathrm{PZTC}$ ligand is deprotonated, which is transformed into $\mathrm{H}_{2} \mathrm{PZTC}^{-}$anion. Furthermore, the $\mathrm{H}_{2} \mathrm{PZTC}^{-}$anion linked $\mathrm{Cu}$ (II) ions into 1D double chain structure. Different from the procedure of $\mathbf{1}$, complex 2 was obtained with two carboxylate groups of $\mathrm{H}_{3}$ PZTC ligand deprotonated under hydrothermal condition with the synthetic temperature of $130{ }^{\circ} \mathrm{C}$ and $\mathrm{pH}$ of 2.2 . In the complex, the $\mathrm{H}_{3}$ PZTC is transformed into HPZTC ${ }^{2-}$ dianion, which coordinates with $\mathrm{Cu}$ (II) ions to form 1D zigzag chain structure. Complex 3 was obtained at $\mathrm{pH}$ value of 3.8 and with the same temperature as that for complex 2 . In 3, $\mathrm{H}_{3} \mathrm{PZTC}$ ligand was converted to 2-hydroxypyrazine3,5-dicarboxylic acid(PZHD) by in situ decarboxylation and hydroxylization reaction. The PZHD ligand further connects the $\mathrm{Cu}(\mathrm{II})$ ions to form 2D layer structure. The results indicate that the formation of complexes 1-3 shows significant dependence on synthesis conditions, such as temperature and $\mathrm{pH}$ value etc. Higher temperature and higher $\mathrm{pH}$ favors in situ $\mathrm{Cu}(\mathrm{II}) / \mathrm{H}_{3} \mathrm{PZTC}$ reaction. In the in situ metal/ligand reaction, the PZHD ligand has not been prepared in organic synthesis field yet. This shows that the in situ metal/ligand reaction can act as a bridge between coordination chemistry and organic synthesis.

In addition, it is noteworthy that both complexes $\mathbf{2}$ and $\mathbf{3}$ have been successfully prepared in the presence of $\mathrm{NaN}_{3}$, but the $\mathrm{NaN}_{3}$ was not coordinated to $\mathrm{Cu}$ (II) ions. When we try to synthesize complexes $\mathbf{2}$ and $\mathbf{3}$ at the same condition except without $\mathrm{NaN}_{3}$, no single-crystal appropriated were obtained. Meanwhile, when we try to synthesize the complexes at the same $\mathrm{pH}$ value, which was adjusted by $\mathrm{NaOH}$ solution, no such product were obtained. Even though the mechanism is not very clear, but this may suggest that $\mathrm{NaN}_{3}$ play a role in adjusting the microenvironment of the system and promote the formation of single-crystals. The mechanism of action deserves further discussion.

Structural Description of $\left[\mathrm{Cu}\left(\mathrm{H}_{2} \mathrm{PZTC}\right)_{2}\right]_{\mathbf{n}} \cdot \mathbf{2} \mathbf{n H}_{2} \mathrm{O}$ (1). The asymmetric unit of $\mathbf{1}$ consists of one half $\mathrm{Cu}(\mathrm{II})$ atom, one partially deprotonated $\mathrm{H}_{2} \mathrm{PZTC}^{-}$anion and one lattice water molecule. The $\mathrm{Cu}(\mathrm{II})$ atom lies on the inversion center

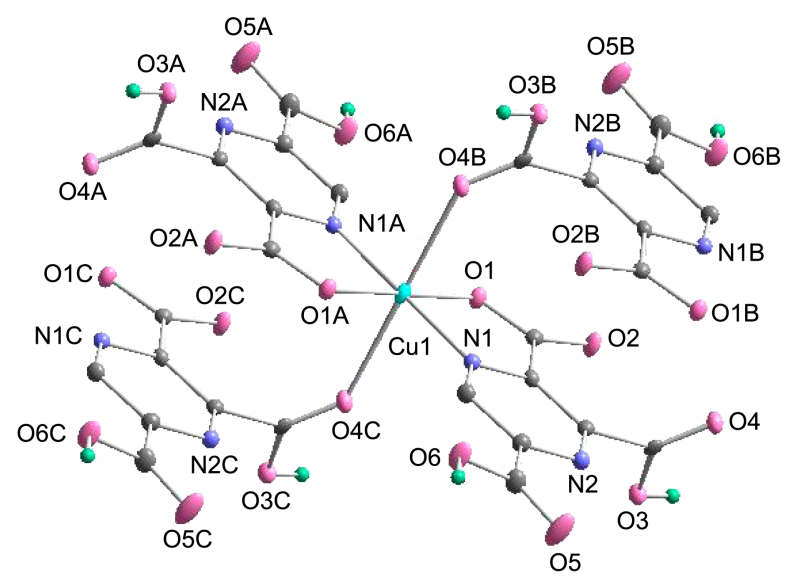

Figure 1. The coordination environment of $\mathrm{Cu}$ (II) ion in $\mathbf{1}$ drawn at $50 \%$ probability level. All non-protonated hydrogen atoms and lattice-water molecules are omitted for clarity. and is coordinated with four $\mathrm{O}$ atoms and two $\mathrm{N}$ atoms (see Fig. 1). The equatorial plane contains four perfectly coplanar atoms, $\mathrm{O} 1$ and $\mathrm{O} 1 \mathrm{~A}[\mathrm{Cu}-\mathrm{O} 1(\mathrm{O} 1 \mathrm{~A})=1.9543(15) \AA]$ from two carboxylate groups of two $\mathrm{H}_{2} \mathrm{PZTC}^{-}$anions, $\mathrm{N} 1$ and $\mathrm{N} 1 \mathrm{~A}[\mathrm{Cu}-\mathrm{N} 1(\mathrm{~N} 1 \mathrm{~A})=1.9931(18) \AA]$ from two $\mathrm{H}_{2} \mathrm{PZTC}^{-}$ anions. $\mathrm{O} 4 \mathrm{~B}$ and $\mathrm{O} 4 \mathrm{C}[\mathrm{Cu}-\mathrm{O} 4 \mathrm{~B}(\mathrm{O} 4 \mathrm{C})=2.3978(15) \AA]$ from two protonated carboxylate groups of two $\mathrm{H}_{2} \mathrm{PZTC}^{-}$ anions occupy the axial sites with the $\mathrm{O} 4 \mathrm{~B}-\mathrm{Cu} 1-\mathrm{O} 4 \mathrm{C}$ angle of $180.0^{\circ}$. Thus, $\mathrm{Cu}(\mathrm{II})$ atom displays distorted octahedral coordination environment.

In complex 1, the $\mathrm{H}_{3} \mathrm{PZTC}$ ligand only looses one proton, which is transformed into $\mathrm{H}_{2} \mathrm{PZTC}^{-}$anion. Each $\mathrm{H}_{2} \mathrm{PZTC}^{-}$ anion adopts tridentate modes to coordinate with two $\mathrm{Cu}$ atoms (see Scheme 2a). Atom O1 of the deprotonated carboxylate group and atom $\mathrm{N} 1$ of pyrazine ring adopt bidentate chelating mode to connect one $\mathrm{Cu}$ atom. Atom $\mathrm{O} 4$ of one protonated carboxylate group links the other $\mathrm{Cu}$ atom. The remaining protonated carboxylate group is free. To the best of our knowledge, this type of coordination mode for $\mathrm{H}_{2} \mathrm{PZTC}^{-}$ligand has not been reported in the literatures.

In this way, each $\mathrm{Cu}$ atom is connected by the $\mathrm{H}_{2} \mathrm{PZTC}^{-}$

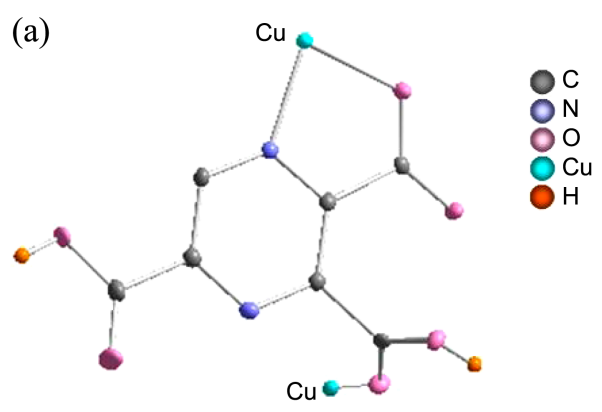

(b)

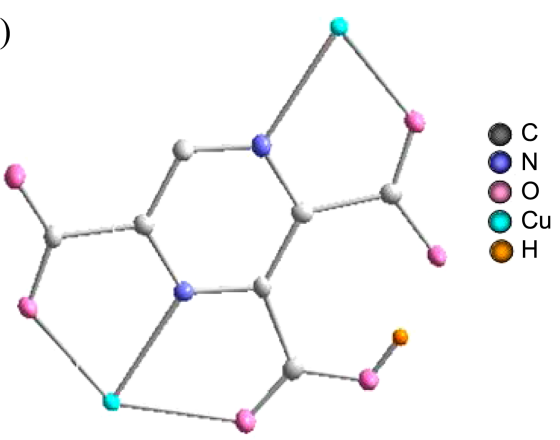

(c)

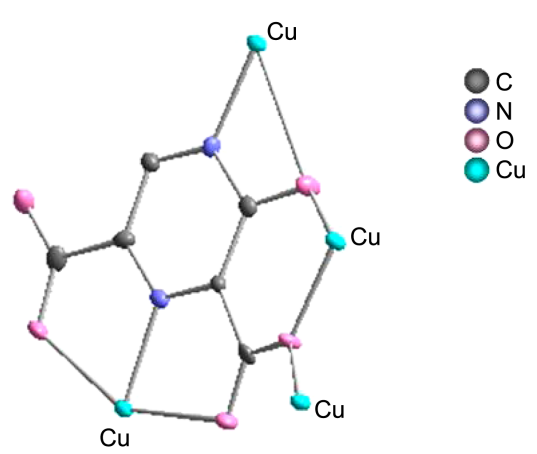

Scheme 2. Coordination modes of the ligands in complexes 1-3. 


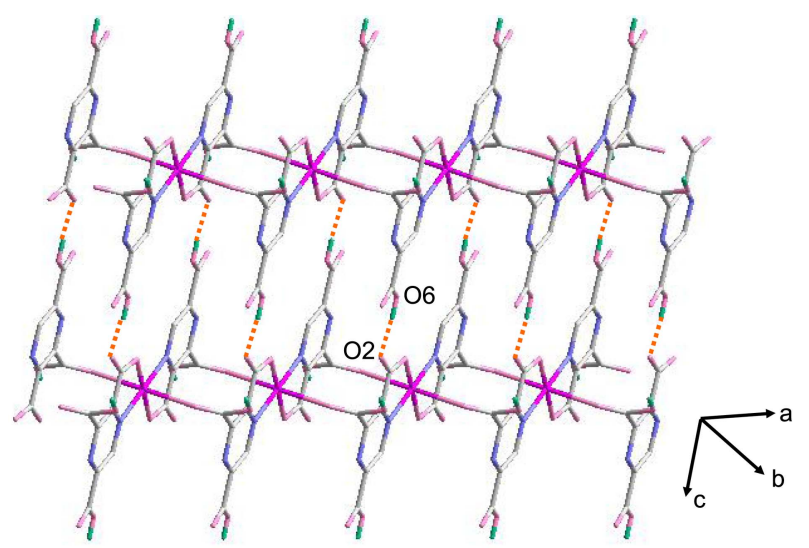

Figure 2. Supramolecular structure of $\mathbf{1}$ containing the hydrogen bonds.

anions to form infinite 1-D double chain along the a-axis direction. These chains are further linked by hydrogen bonds $\left(\mathrm{O} 6-\mathrm{H} 6 \cdots \mathrm{O} 2,2.554(2) \AA, 171.9(2)^{\circ}\right)$ with protonated carboxylate groups as donors and deprotonated carboxylate groups as acceptors into 2D supramolecular network along the a-axis (see Fig. 2). Free water molecules reside between the layers and involve in the formation of hydrogen bonds between the protonated carboxylate groups and the uncoordinated nitrogen atom, which further build up the three-dimensional supramolecular network.

Structural Description of $\left[\mathrm{Cu}(\mathrm{HPZTC}) \cdot 2 \mathrm{H}_{2} \mathrm{O}\right]_{n} \cdot 2 \mathbf{n H}_{2} \mathrm{O}$ (2). The asymmetric unit of $\mathbf{2}$ consists of one $\mathrm{Cu}$ (II) atom, one $\mathrm{HPZTC}^{2-}$ dianion and two coordinated water molecules together with two lattice water molecules. $\mathrm{Cu}(\mathrm{II})$ atom is seven-coordinated with a $\mathrm{N}_{2} \mathrm{O}_{5}$ donor set and displays distorted pentagonal bipyramidal geometry (see Fig. 3). Five basal positions are filled with N1, O1, O6, N2A and O3A atoms from two $\mathrm{HPZTC}^{2-}$ dianions. The $\mathrm{Cu}-\mathrm{O}$ bond lengths are in the range of 2.1939 (17) to 2.3012(17) $\AA$. The $\mathrm{Cu}-\mathrm{N}$ bond distances are 2.241(2) $\AA$ and 2.397(2) $\AA$. The axial sites are filled with two aqua oxygen atoms ( 07 and $\mathrm{O} 8)$, the $\mathrm{Cu}-\mathrm{O}$ distances are 2.0271(16) $\AA$ and 2.0329(16) $\AA$, the angle of $\mathrm{O} 7-\mathrm{Cu} 1-\mathrm{O} 8$ is $173.30(7)^{\circ}$.

Different from 1, HPZTC ${ }^{2-}$ dianion in $\mathbf{2}$ is formed from $\mathrm{H}_{3}$ PZTC by loosing two protons. Each HPZTC ${ }^{2-}$ dianion links two $\mathrm{Cu}$ atoms (see Scheme $2 \mathrm{~b}$ ) in pentadentate modes:

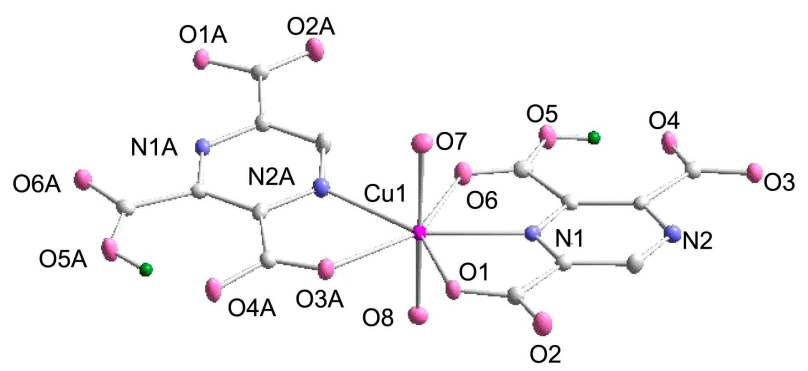

Figure 3. The coordination environment of $\mathrm{Cu}(\mathrm{II})$ ion in $\mathbf{2}$ drawn at $50 \%$ probability level. All hydrogen atoms except the not deprotonated (green ball) and lattice-water molecules are omitted for clarity.
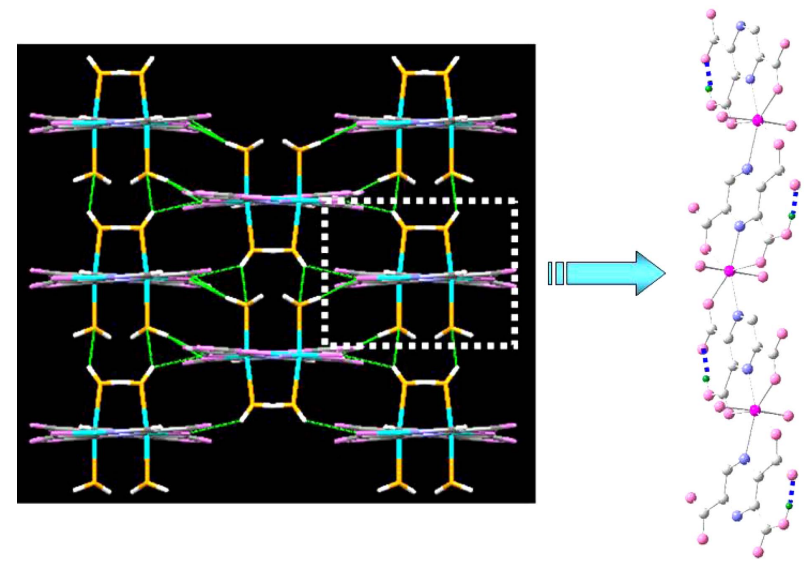

Figure 4. 3D supramolecular network of 2 viewed along the c-axis direction.

pyrazine nitrogen atom (N1) and two neighboring oxygen atoms (O1 and O6) of carboxylate groups connect one $\mathrm{Cu}$ atom, the other nitrogen atom $(\mathrm{N} 2)$ of pyrazine ring and oxygen atom (O3) of the third carboxylate group link the second $\mathrm{Cu}$ atom. Thus, a one-dimensional (1D) polymeric zigzag chain is formed along c-axis direction. In the $1 \mathrm{D}$

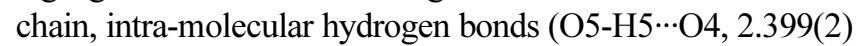
$\left.\AA, 168.0(5)^{\circ}\right)$ exist between protonated and deprotonated neighboring carboxylate groups. Each 1D zigzag chain is further linked with six neighbors via hydrogen bonds (O7H7B $\cdots \mathrm{O} 8,3.015(2) \AA, 138.7(2) ; \mathrm{O} 7-\mathrm{H} 7 \mathrm{~B} \cdots \mathrm{O} 3,2.881(2) \AA$, 130.1(2) ${ }^{\circ}$; O8-H8A $\left.\cdots \mathrm{O} 1,2.729(2) \AA, 163.2(2)^{\circ}\right)$ between coordinated water molecules and oxygen atoms of carboxylate groups into 3D supramolecular network containing small 1D channels viewed along c-axis (see Figure 4). The free water molecules reside in channel spaces and form hydrogen bonds with the carboxylate groups and coordinated water molecules, which further stabilize the structure.

Structural Description of $\left[\mathrm{Cu}_{2}\left[(\mathrm{PZHD})(\mathrm{OH})\left(\mathrm{H}_{2} \mathrm{O}\right)_{2}\right]_{\mathbf{n}}\right.$ (3). Asymmetric unit of complex 3 consists of two crystallographically independent $\mathrm{Cu}$ atoms, one PZHD ligand, two coordinated water molecules and one hydroxyl group. The coordination environments of $\mathrm{Cu}$ atoms are shown in Figure

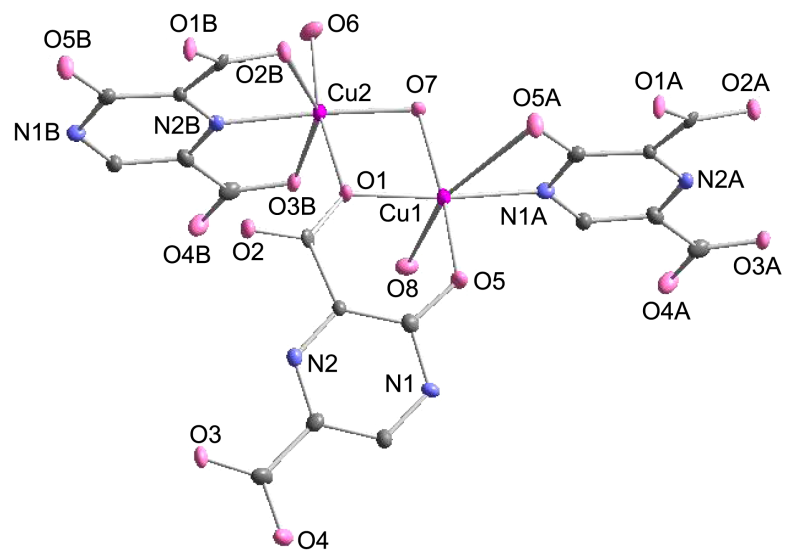

Figure 5. The coordination environments of $\mathrm{Cu}(\mathrm{II})$ ions in $\mathbf{3}$ drawn at $50 \%$ probability level. All hydrogen atoms and lattice-water molecules are omitted for clarity. 
5. Cul atom is six-coordinated with a NO5 donor set; the equatorial plane contains atoms $\mathrm{O} 1, \mathrm{O} 5, \mathrm{~N} 1 \mathrm{~A}[\mathrm{Cu} 1-\mathrm{O} 1=$ 1.983(3) $\AA, \mathrm{Cu} 1-\mathrm{O} 5=1.921(4) \AA, \mathrm{Cu} 1-\mathrm{N} 1 \mathrm{~A}=1.994(4) \AA]$ from two PZHD ligands, and O7 $[\mathrm{Cu} 1-\mathrm{O} 7=1.916(4) \AA]$ from hydroxyl group. Atoms $\mathrm{O} 8$ and O5A [Cu1-O8 = 2.493(4) and $\mathrm{Cu} 1-\mathrm{O} 5 \mathrm{~A}=2.706(4) \AA]$ occupy the axial sites, which from water molecule and carboxylate group of PZHD ligand with the O8-Cu1-O5A angle of $155.41(13)^{\circ}$. Thus, the coordination environment of $\mathrm{Cu} 1$ can be described as distorted octahedron. $\mathrm{Cu} 2$ atom is also six-coordinated with a NO5 donor set, and displays distorted octahedral geometry, but the coordination environment is different. The basal plane contains atoms $\mathrm{O} 2 \mathrm{~B}, \mathrm{O} 3 \mathrm{~B}$ and $\mathrm{N} 2 \mathrm{~B}[\mathrm{Cu} 2-\mathrm{O} 2 \mathrm{~B}$ $=2.382(4) \AA, \mathrm{Cu} 2-\mathrm{O} 3 \mathrm{~B}=2.383(4) \AA, \mathrm{Cu} 2-\mathrm{N} 2 \mathrm{~B}=2.014(4)$ $\AA]$ from the same one PZHD ligand, and atom O7 [Cu2-O7 $=1.937(3) \AA]$ from hydroxyl group. The axial sites are occupied by atoms $\mathrm{O} 1$ and $\mathrm{O} 6[\mathrm{Cu} 2-\mathrm{O} 1=2.058(4)$ and $\mathrm{Cu} 2-\mathrm{O} 6=1.957(4) \AA]$ from carboxylate group of the other PZHD ligand and one water molecule. The angle of O6$\mathrm{Cu} 2-\mathrm{O} 1$ is $169.18(15)^{\circ}$.

In complex 3, each PZHD ligand adopts octadentate mode to bind four $\mathrm{Cu}$ atoms, as shown in Scheme $2 \mathrm{c}$ : one pyrazine nitrogen atom together with two adjacent carboxylate oxygen atoms coordinate one $\mathrm{Cu}(\mathrm{II})$ atom, the other one pyrazine nitrogen atom and the adjacent hydroxyl group oxygen atom coordinate the second $\mathrm{Cu}$ (II) atom. Simultaneously, the hydroxyl group oxygen atom and the adjacent carboxylate group oxygen atom link the third $\mathrm{Cu}$ (II) atom, the same carboxylate oxygen atom link the fourth $\mathrm{Cu}$ (II) atom. This kind of coordination mode for PZHD ligand is different from our previously reported. ${ }^{21}$ As a result, PZHD ligands connect the $\mathrm{Cu}(\mathrm{II})$ atoms into $2 \mathrm{D}$ layer structure (see Fig. 6).

In the 2D layer structure, there are two different rings, one is 4-membered ring containing the $\left\{\mathrm{Cu}_{2} \mathrm{O}_{2}\right\}$ unit. In the $\left\{\mathrm{Cu}_{2} \mathrm{O}_{2}\right\}$ unit, $\mathrm{Cu}$ (II) atoms are linked by carboxylate $\mu_{2}-\mathrm{O}$ bridge and $\mu_{2}-\mathrm{OH}$ bridge, the $\mathrm{Cu}-\mathrm{Cu}$ separation is 3.015(12) $\AA$. The other is the 14-membered ring containing five $\mathrm{Cu}$ atoms. Furthermore, these layers are assembled via hydrogen bonds into 3D supramolecular network.

Thermal Stability Analyses. In order to test the thermal stability of the title complexes $\mathbf{1 - 3}$, the thermogravimetric analysis of them were carried out. For complex 1, the thermal decomposition process shows two steps: the first weight loss of $7.22 \%$, which occurred between 49 and 108

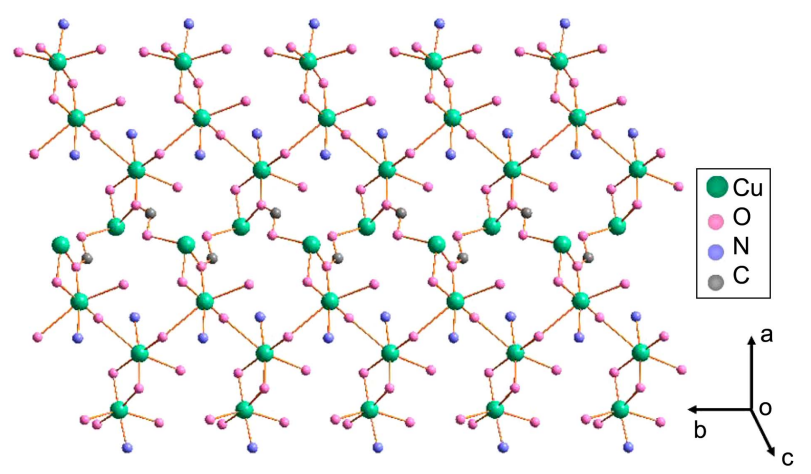

Figure 6. The 2D metal-organic framework of 3.
${ }^{\circ} \mathrm{C}$, corresponds to the release of two water molecules (calculated: 6.70\%). As temperature rose, no obvious plateau appeared indicating that complex $\mathbf{1}$ continued to decompose and finally $\mathrm{CuO}$ power was obtained with $16.03 \%$ (calculated: $15.90 \%$ ).

For complex 2, the thermal decomposition process displays three steps: the first weight loss of $13.33 \%$ occurring between 146 and $212{ }^{\circ} \mathrm{C}$ may correspond to the release of two uncoordinated water molecules and 0.5 coordinated water molecules (calculated: $13.02 \%$ ). And then the second weight loss of $34.82 \%$ (calcd $33.27 \%$ ) between 262 and 315 ${ }^{\circ} \mathrm{C}$ may correspond to release of the remaining 1.5 coordinated water molecules and two carboxylate groups. The framework collapsed rapidly above the temperature $315{ }^{\circ} \mathrm{C}$.

For complex 3, a complicated decomposition reaction takes place. The first weight loss started at $108^{\circ} \mathrm{C}$, as temperature rose, decomposition continued and no obvious plateau appeared, which indicates that not strong boundaries between the losing water molecules and PZHD ligands. This shows that hydrogen bonds play an important role in the assembly of the 3D supramolecular network from 2D metalorganic framework.

\section{Conclusions}

Three new copper complexes were synthesized from $\mathrm{Cu}(\mathrm{II}) / \mathrm{H}_{3} \mathrm{PZTC}$ system under different synthetic conditions, and characterized by single-crystal X-ray diffraction. Structure analyses of the title complexes have show that three carboxylate groups of $\mathrm{H}_{3} \mathrm{PZTC}$ ligand are very sensitive to $\mathrm{pH}$ values, and can partially or fully deprotonate under different $\mathrm{pH}$ values. In complexes 1 and 2, $\mathrm{H}_{3}$ PZTC ligands loose one and two protons at $\mathrm{pH}=1.8$ and 2.2 , respectively. Such behavior of deprotonation at different acidity can help us to investigate the $\mathrm{pH}$ effect on the formation of coordination networks. In complex $\mathbf{3}, \mathrm{H}_{3}$ PZTC ligand converted into PZHD by in situ decarboxylation and hydroxylization reaction under hydrothermal condition with the synthetic temperature at 130 and $\mathrm{pH}=3.3$. The study on $\mathrm{Cu} / \mathrm{H}_{3}$ PZTC system has shown that the final reaction product is significantly affected by the preparation conditions.

Supplementary Materials. Crystallographic data for the structures reported in this paper have been deposited at the Cambridge Crystallographic Data Center as supplementary publication: CCDC Nos. 759021-759023 for 1-3. Copies of the data can be obtained free of charge on application to CCDC, 12 Union Road, Cambridge CB2 1EZ, UK (fax: +44 1223336 033; e-mail: deposit@ccdc.cam.ac.uk or http:// www.ccdc.cam.ac.uk).

Acknowledgments. We thank Tianjin Polytechnic University for financial support.

\section{References}

1. Dybtsev, D. N.; Nuzhdin, A. L.; Chun, H.; Bryliakov, K. P.; Talsi, 
E. P.; Fedin, V. P.; Kim, K. Angew. Chem., Int. Ed. 2006, 45, 916.

2. Gao, C. Y.; Liu, S. X.; Xie, L. H.; Sun, C. Y.; Cao, J. F.; Ren, Y. H.; Feng, D.; Su, Z. M. CrystEngComm. 2009, 11, 177.

3. Liu, C. M.; Zuo, J. L.; Zhang, D. Q.; Zhu, D. B. CrystEngComm. 2008, 10,1674

4. Li, C. J.; Peng, M. X.; Leng, J. D.; Yang, M. M.; Lin, Z. J.; Tong, M. L. CrystEngComm. 2008, 10, 1645.

5. Chen, B. L.; Wang, L. B.; Zapata, F.; Qian, G. D.; Lobkovsky, E. B. J. Am. Chem. Soc. 2008, 130, 6718 .

6. Xue, M.; Liu, Y.; Schaffino, R. M.; Xiang, S. C.; Zhao, X. J.; Zhu, G. S.; Qiu, S. L.; Chen, B. L. Inorg. Chem. 2009, 48, 4649.

7. Li, Y. W.; Yang, R. T. Langmuir 2007, 23, 12937.

8. Bauer, C. A.; Timofeeva, T. V.; Settersten, T. B.; Patterson, B. D.; Liu, V. H.; Simmons, B. A.; Allendorf, M. D. J. Am. Chem. Soc. 2007, 129, 7136 .

9. Kupplera, R. J.; Timmons, D. J.; Fang, Q. R.; Li, J. R.; Makal, T. A.; Young, M. D.; Yuan, D. Q.; Zhao, D.; Zhuang, W. J.; Zhou, H. C. Coord. Chem. Rev. 2009, 253, 3042.

10. Férey, G. Chem. Soc. Rev. 2008, 37, 191

11. Binnemans, K. Chem. Rev. 2009, 109, 4283.

12. Lan, A. J.; Li, K. H.; Wu, H. H.; Olson, D. H.; Emge, T. J.; Ki, W.; Hong, M. C.; Li, J. Angew. Chem. Int. Ed. 2009, 48, 2334.

13. Chen, J. X.; Ohba, M.; Zhao, D. Y.; Kaneko, W.; Kitagawa, S. Cryst. Growth \& Des. 2006, 6, 664.

14. Wang, H.; Wang, Y. Y.; Yang, G. P.; Wang, C. J.; Wen, G. L.; Shi, Q. Z.; Batten, S. R. CrystEngComm. 2008, 10, 1583.

15. Zhang, J. P.; Lin, Y. Y.; Zhang, W. X.; Chen, X. M. J. Am. Chem.
Soc. 2005, 127, 14162.

16. Pan, L.; Frydel, T.; Sander, M. B.; Huang, X. Y.; Li, J. Inorg. Chem. 2001, 40, 1271.

17. Go, Y. B.; Wang, X. X.; Anokhina, E. V.; Jacobson, A. J. Inorg Chem. 2005, 44, 8265.

18. Yu, Q.; Zhang, X. Q.; Bian, H. D.; Liang, H.; Zhao, B.; Yan, S. P.; Liao, D. Z. Cryst. Growth \& Des. 2008, 8, 1140.

19. Dai, J. C.; Wu, X. T.; Fu, Z. Y.; Cui, C. P.; Hu, S. M.; Du, W. X.; Wu, L. M.; Zhang, H. H.; Sun, R. O. Inorg. Chem. 2002, 41, 1391.

20. Pan, Z. R.; Zheng, H. G.; Wang, T. W.; Song, Y.; Li, Y. Z.; Guo, Z. J.; Batten, S. R. Inorg. Chem. 2008, 47, 9528.

21. Wang, F. Q.; Mu, W. H.; Zheng, X. J.; Li, L. C.; Fang, D. C.; Jin, L. P. Inorg. Chem. 2008, 47, 5225.

22. Wan, Y. H.; Zheng, X. J.; Wang, F. Q.; Zhou, X. Y.; Wang, K. Z.; Jin, L. P. CrystEngComm. 2009, 11, 278.

23. Mager, H. I. X.; Berends, W. Recl. Trav. Chim. Pays-Bas 1958, $77,827$.

24. Weygand, F. Chem. Ber. 1940, 73, 1259.

25. Weygand, F.; Bergmann, C. Chem. Ber. 1947, 80, 255.

26. Sheldrick, G. M. SADABS, Program for Empirical Absorption Correction of Area Detector Data, University of Göttingen: Göttingen, Germany, 1997.

27. Sheldrick, G. M. SHELXS 97, Program for Crystal Structure Solution, University of Göttingen, Göttingen, Germany, 1997.

28. Sheldrick, G. M. SHELXL 97, Program for Crystal Structure Refinement, University of Göttingen, Göttingen, Germany, 1997. 